\title{
Structure evolution in electrorheological and magnetorheological suspensions from a continuum perspective
}

\author{
Karl von Pfeil, Michael D. Graham, and Daniel J. Klingenberga) \\ Department of Chemical Engineering, University of Wisconsin, 1415 Engineering Drive, Madison, \\ Wisconsin 53706 \\ Jeffrey F. Morris \\ School of Chemical Engineering, Georgia Institute of Technology, Atlanta, Georgia 30332-0100
}

(Received 19 September 2002; accepted 31 January 2003)

\begin{abstract}
A two-fluid continuum model is developed to describe mass transport in electro- and magnetorheological suspensions. The particle flux is related to the field-induced and hydrodynamic stresses. Solutions of the resulting mass balance show column formation in the absence of flow and stripe formation when a suspension is subjected simultaneously to an applied electric field and shear flow. (C) 2003 American Institute of Physics. [DOI: 10.1063/1.1563037]
\end{abstract}

\section{INTRODUCTION}

Electro- and magnetorheological (ER and MR) fluids are particulate suspensions whose rheological properties are dramatically altered by electric and magnetic fields, respectively. ${ }^{1-4}$ In shear flow, applied fields can increase the apparent viscosity by several orders of magnitude. This phenomenon is now being exploited in commercial applications. ${ }^{5,6}$

Along with the rheological phenomena, the applied fields also alter the suspension microstructure. In quiescent suspensions, applied fields cause the formation of particulate columns oriented in the direction of the field. ${ }^{2,4}$ In shear flow, applied fields cause the formation of concentrated particulate stripes oriented in the flow direction. ${ }^{7-11}$ These patterns are intimately connected to the rheological phenomena.

Particle-level models and simulations have been valuable for understanding the relationships between particle properties, interactions, and macroscopic behavior. Simulations employing dipolar particle interactions commonly report the field-induced formation of fibrous aggregates in quiescent suspensions, as well as the appearance of an apparent yield stress directly related to the particles' polarizability. ${ }^{4,12}$ Melrose and Heyes ${ }^{13}$ and Martin ${ }^{14}$ observed the formation of layered structures in simulations of simple shear flow, similar to the stripe structures observed experimentally. Unfortunately, simulation techniques are limited to small numbers of particles and, hence, are not well suited for describing phenomena involving large numbers of particles or predicting the behavior of a suspension throughout an entire device. These issues are particularly important in situations where the field, temperature, or particle concentration are not uniform.

In a previous article, we presented a continuum description of the evolution of the structure of ER suspensions, ${ }^{15}$ as characterized by the time- and position-dependent particle volume fraction $\phi(\mathbf{x}, t)$. This was accomplished by develop-

a) Author to whom correspondence should be addressed. Electronic mail: klingen@engr.wisc.edu ing a conservation equation for the particle concentration, using an expression for the particle flux obtained from a momentum balance. The particle flux is related to the divergence of the particle contribution to the stress. Solutions of this conservation equation were obtained by accounting only for the electrostatic contribution to the stress, ignoring the hydrodynamic contribution. Using this two-fluid approach, one predicts the patterns commonly observed experimentally. For electric fields applied to quiescent suspensions, this approach captures the formation of particulate columns; for electric fields applied to flowing suspensions, it captures the formation of particulate stripes oriented in the flow direction. A similar analysis for MR fluids follows directly.

In this article we revisit this continuum analysis of the evolution of the particle volume fraction in ER suspensions in simple shear flow, accounting for both the electrostatic and hydrodynamic contributions to the stress. At small shear rates, the stability of a uniform distribution of particles is unaffected by the hydrodynamic contribution to the stress, and again one predicts the formation of stripes in sheared suspensions. At sufficiently large shear rates, the hydrodynamic contribution to the stress dominates and the uniform concentration profile remains stable (although the model must be slightly modified to achieve stability in this limit, as described in the text). The stability analysis suggests that the particle volume fraction evolves to a function of only time and position in the vorticity direction, motivating the development of a one-dimensional model for particle transport [i.e., $\phi=\phi(y, t)$ where $y$ is the vorticity direction]. This simplification provides the same stability criterion and also permits the solution of the steady-state concentration profile. For conditions where stripes are formed at steady state, the concentration within the stripes is determined by the balance of electrostatic and hydrodynamic contributions to the stress. Finally, we show that nonlocal contributions to the electrostatic contribution to the stress do not alter the stability criteria, but do affect the size and growth rates of the structures formed. 


\section{TWO-FLUID MODEL FOR MASS TRANSPORT}

The flow model is a generalization to ER and MR fluids of that presented by Morris and Boulay ${ }^{16}$ for noncolloidal suspensions in shear flows, which is related to the earlier work of Nott and Brady. ${ }^{17}$ Here, we present a brief outline of the derivation of the equation governing particle transport, with details available from these references. For typical applications, the flow at the particle scale in MR and ER fluids is at small Reynolds number and we limit consideration to this case.

The mass conservation equation for the particles is obtained by an averaging of the general mass conservation equation for the mixture,

$$
\frac{\partial \phi}{\partial t}+\langle\mathbf{u}\rangle \cdot \nabla \phi=-\nabla \cdot \mathbf{j}
$$

where

$$
\mathbf{j}=\phi(\mathbf{U}-\langle\mathbf{u}\rangle)
$$

is the difference between the total particle flux and that arising from pure convection with the bulk averaged velocity, which is denoted $\langle\mathbf{u}\rangle$. Determining the average particle velocity $\mathbf{U}$ requires consideration of the momentum balance for the particle phase. Obtained by an averaging procedure over the general momentum equation, for small Reynolds numbers, the particle phase momentum balance is

$$
0=\nabla \cdot \boldsymbol{\sigma}^{(p)}+n \mathbf{F}^{H}+\phi\left(\rho_{p}-\langle\rho\rangle\right) \mathbf{g},
$$

where $\boldsymbol{\sigma}^{(p)}$ is the particle contribution to the bulk stress, $n$ $=\phi /\left(4 \pi a^{3} / 3\right)$ is the local number density of monodisperse particles of radius $a, \mathbf{F}^{H}$ is the average hydrodynamic force per particle, and $\rho_{p}-\langle\rho\rangle$ is the difference in density between the particulate material and the bulk mixture. The hydrodynamic force results from relative motion between the particles and the bulk mixture, and thus may be expressed

$$
\begin{aligned}
n \mathbf{F}^{H} & =-n\langle\mathbf{R} \cdot(\mathbf{U}-\langle\mathbf{u}\rangle)\rangle \\
& \approx-\frac{9 \eta_{c}}{2 a^{2}} \phi f^{-1}(\phi)(\mathbf{U}-\langle\mathbf{u}\rangle) .
\end{aligned}
$$

The first form expresses the hydrodynamic force in terms of a $\phi$-dependent resistance tensor $\mathbf{R}(\phi)$. In place of this resistance tensor approach, a simple model using the sedimentation hindrance function $f(\phi)$ is used. The function $f(\phi)$ is the ratio of the mean sedimentation velocity in a uniform suspension at volume fraction $\phi$ to the Stokes settling velocity. At low Reynolds number, $f(\phi)=(1-\phi)^{\alpha}$ with $\alpha=2-5$ is a typical form (see Ref. 18). Following prior modeling, ${ }^{16}$ $\alpha=4$ is used in this work. Although the chainlike structures in ER and MR suspensions are anisotropic, this isotropic hydrodynamic model is reasonable for the problems considered here-namely, the initial stages of structure formation from an initially isotropic suspension (Secs. II B and II C 1) - as well as the steady-state structure where the relative particle flux is zero (Sec. II C 2).

For neutrally buoyant particles, the particle momentum equation reduces to a balance between the stress divergence and hydrodynamic forces, the latter of which may be expressed in terms of the particle flux via Eqs. (2) and (4b). The migration flux is thus given by

$$
\mathbf{j}=\frac{2 a^{2}}{9 \eta_{c}} f(\phi) \nabla \cdot \boldsymbol{\sigma}^{(p)} .
$$

Inserting this result into Eq. (1) produces an evolution equation for $\phi$ which depends upon the particle stress. In general, this equation must be augmented by the equations governing the bulk suspension flow, given for these conditions simply by $\nabla \cdot\langle\mathbf{u}\rangle=0$ and $\nabla \cdot\langle\boldsymbol{\sigma}\rangle=0$. Here, however, the bulk motion is known because we consider a flow which remains simple shear flow. Of particular interest in this study are the combined effects of electrostatic and hydrodynamic contributions to the particle stress on the evolution of the suspension structure. Modeling of both of these contributions is described below.

We consider the suspension to be composed of dielectric spheres immersed in a dielectric suspending fluid (conducting spheres in a weakly conducting fluid are treated analogously). The suspension structure is assumed to be isotropic and amorphous. Such a structure is reasonable for dilute quiescent or flowing suspensions prior to the application of an electric field, as well as for the initial stages of structure formation following the application of an electric field. For such a suspension, the electrostatic stress is

$$
\boldsymbol{\sigma}^{(E)}=\epsilon_{0}\left[\left(\epsilon-\frac{1}{2} a_{1}\right) \mathbf{E E}-\frac{1}{2}\left(\epsilon+a_{2}\right) E^{2} \boldsymbol{\delta}\right],
$$

where $\epsilon_{0}=8.8542 \times 10^{-12} \mathrm{~F} / \mathrm{m}$ is the permittivity of free space, and $\epsilon, a_{1}$, and $a_{2}$ are described as follows. A selfconsistent mean-field analysis in the point-dipole limit yields a suspension dielectric constant ${ }^{19}$

$$
\epsilon(\phi)=\epsilon_{c} \frac{1+2 \beta \phi}{1-\beta \phi},
$$

where $\epsilon_{c}$ is the dielectric constant of the suspending fluid, $\beta=\left(\epsilon_{p}-\epsilon_{c}\right) /\left(\epsilon_{p}+2 \epsilon_{c}\right)$, and $\epsilon_{p}$ is the dielectric constant of the particulate material. Assuming that the disperse and continuous phases themselves are not electrostrictive, the electrostriction coefficients of the suspension, $a_{1}$ and $a_{2}$, are ${ }^{19}$ $a_{1}=0$ and

$$
a_{2}(\phi)=-\frac{\left[\epsilon(\phi)-\epsilon_{c}\right]\left[\epsilon(\phi)+2 \epsilon_{c}\right]}{3 \epsilon_{c}} .
$$

The particle contribution to the electrostatic stress for this system is

$$
\begin{aligned}
\boldsymbol{\sigma}^{(p, E)} & =\boldsymbol{\sigma}^{(E)}-\boldsymbol{\sigma}^{(E)}(\phi=0) \\
& =\epsilon_{0}\left[\left(\epsilon-\epsilon_{c}\right) \mathbf{E E}-\frac{1}{2}\left(\epsilon-\boldsymbol{\epsilon}_{c}+a_{2}\right) E^{2} \boldsymbol{\delta}\right] .
\end{aligned}
$$

Taking the divergence of Eqs. (6) and (10) and utilizing Maxwell's equations $\nabla \cdot \mathbf{D}=\rho^{(e)}\left(\rho^{(e)}\right.$ is the free charge density, which is zero for the present analysis) and $\nabla \times \mathbf{E}=\mathbf{0}$ gives

$$
\begin{aligned}
\nabla \cdot \boldsymbol{\sigma}^{(p, E)} & =\nabla \cdot \boldsymbol{\sigma}^{(E)} \\
& =-\frac{1}{2} \epsilon_{0} E^{2} \nabla \epsilon-\frac{1}{2} \epsilon_{0} \nabla\left(a_{2} E^{2}\right) .
\end{aligned}
$$


The terms on the right side of the last equation are body forces. Since the divergences of $\boldsymbol{\sigma}^{(E)}$ and $\boldsymbol{\sigma}^{(p, E)}$ are equal, these body forces are exerted on the particles.

Morris and Boulay ${ }^{16}$ investigated the hydrodynamic stress in sheared, noncolloidal, hard-sphere suspensions. In such systems, the stress tensor contains anisotropic normal stress contributions. Morris and Boulay write the particle contribution to the hydrodynamic stress,

$$
\boldsymbol{\sigma}^{(p, H)}=-\eta_{n} \hat{\mathbf{Q}} \dot{\gamma}+\eta_{p}\left[\nabla\langle\mathbf{u}\rangle+(\nabla\langle\mathbf{u}\rangle)^{T}\right],
$$

where the first term represents the shear-induced normal stresses. Here $\eta_{n}(\phi)$ is the "normal stress viscosity," and $\eta_{p}(\phi)$ is the particle contribution to the shear viscosity [i.e., the suspension shear viscosity is $\left.\eta_{s}(\phi)=\eta_{c}+\eta_{p}(\phi)\right]$. With the $x, y$, and $z$ directions (or the 1,2 , and 3 directions) oriented with the flow, vorticity, and gradient directions, respectively, the normal stresses are specified by the tensor $\mathbf{Q}$ given by

$$
\hat{\mathbf{Q}}=\left(\begin{array}{ccc}
1 & 0 & 0 \\
0 & \lambda_{2} & 0 \\
0 & 0 & \lambda_{3}
\end{array}\right),
$$

where $\lambda_{2} \cong 0.5$ and $\lambda_{3} \cong 0.8$ provide best fits of various suspension flows. These values are used in this article.

The particle contributions to the shear and normal stress viscosities were treated empirically as

$\eta_{p}=2.5 \eta_{c} \phi\left(1-\frac{\phi}{\phi_{\max }}\right)^{-1}+K_{s} \eta_{c}\left(\frac{\phi}{\phi_{\max }}\right)^{2}\left(1-\frac{\phi}{\phi_{\max }}\right)^{-2}$,

$\eta_{n}=K_{n} \eta_{c}\left(\frac{\phi}{\phi_{\max }}\right)^{2}\left(1-\frac{\phi}{\phi_{\max }}\right)^{-2}$,

where $\phi_{\max }$ is the maximum packing fraction $\left(\phi_{\max }=0.68\right.$ in this study), and $K_{s}=0.1$ and $K_{n}=0.75$ provided reasonable agreement with experimental data.

Utilizing the above descriptions of the particle contribution to the stress, we can express the flux relative to the mean flow in an electrified suspension as

$$
\mathbf{j}=\frac{2 a^{2}}{9 \eta_{c}} f(\phi) \nabla \cdot\left(\boldsymbol{\sigma}^{(p, E)}+\boldsymbol{\sigma}^{(p, H)}\right),
$$

where $\boldsymbol{\sigma}^{(p, E)}$ and $\boldsymbol{\sigma}^{(p, H)}$ are given by Eqs. (10) and (13), respectively. Substituting this result into Eq. (1) gives, for the particle conservation equation,

$$
\frac{\partial \phi}{\partial t}+\langle\mathbf{u}\rangle \cdot \nabla \phi=-\frac{2 a^{2}}{9 \eta_{c}} \nabla \cdot\left[f(\phi) \nabla \cdot\left(\boldsymbol{\sigma}^{(p, E)}+\boldsymbol{\sigma}^{(p, H)}\right)\right] .
$$

This equation along with Maxwell's equations provides a complete description of the concentration distribution for cases in which the suspension average velocity profile $\langle\mathbf{u}\rangle$ is known.

In the following sections, we investigate the implications of this model. In Sec. II A, we consider dielectrophoresis in a uniform suspension. The particle flux in a nonuniform field predicted by the model developed above agrees with that predicted with a conventional microscopic analysis. In Sec.
II B, the linearized version of Eq. (18) is investigated in order to determine the early stages of structure evolution following the application of an electric field to a suspension. For quiescent suspensions, this model predicts the formation of particle-rich columns, as commonly observed. For a suspension exposed simultaneously to simple shear flow and an electric field, the two-fluid model predicts the formation of particle-rich stripes oriented in the flow direction, again as commonly observed. Incorporating hydrodynamic interactions has little impact on the resulting structures, at least at small shear rates. These results suggest that the suspension structure under shear varies only in the vorticity direction. We thus consider a one-dimensional model of structure evolution in Sec. II C, where we determine the steady-state stripe structure as a function of field strength and shear rate. In Sec. II D, we examine some effects of nonlocal polarization on particle transport and show that nonlocal polarization does not alter the types of structures formed, but does alter the formation rates and the characteristic length scales. Conclusions from this study are summarized in Sec. III.

\section{A. Dielectrophoresis}

Here we consider the behavior of a quiescent suspension of uniform concentration $\phi$ subjected to a nonuniform electric field. The applied field will generate a dipole moment on each particle; the nonuniform field will produce a force on each particle and, thus, a particle flux. This phenomenon is known as dielectrophoresis. We show that the flux predicted by the two-fluid model is equivalent to that predicted by a traditional microscopic approach.

The nonuniformity of the applied electric field is assumed to be sufficiently weak such that the positiondependent field may be written

$$
\mathbf{E}(\mathbf{x})=\mathbf{E}_{0}+\mathbf{x} \cdot(\nabla \mathbf{E})_{0},
$$

where $\mathbf{E}_{0}$ and $(\nabla \mathbf{E})_{0}$ are the electric field and field gradient at the origin, respectively.

Consider first a microscopic description of the particle dynamics. The dipole moment on a sphere at the origin is given by ${ }^{19}$

$$
\mathbf{p}=4 \pi \boldsymbol{\epsilon}_{0} \boldsymbol{\epsilon}_{c} a^{3} \beta \mathbf{E}_{0} .
$$

This particle will experience a force given by ${ }^{20}$

$$
\mathbf{F}=\mathbf{p} \cdot(\nabla \mathbf{E})=4 \pi \epsilon_{0} \epsilon_{c} a^{3} \beta \mathbf{E}_{0} \cdot(\nabla \mathbf{E})_{0} .
$$

The particle velocity is simply $\mathbf{u}=\mathbf{F} / 6 \pi \eta_{c} a$, and thus the particle flux, $\mathbf{j}=\phi \mathbf{u}$ is

$$
\mathbf{j}=\frac{2 \epsilon_{0} \epsilon_{c} a^{2} \beta}{3 \eta_{c}} \mathbf{E}_{0} \cdot(\nabla \mathbf{E})_{0} \phi .
$$

Next consider the flux predicted by the two-fluid approach. Substituting Eq. (19) into Eq. (12) and utilizing Eq. (8) for $a_{2}(\phi)$ yields, in the limit of small particle concentrations,

$$
\nabla \cdot \boldsymbol{\sigma}^{(p, E)}=3 \epsilon_{0} \epsilon_{c} \beta \mathbf{E}_{0} \cdot(\nabla \mathbf{E})_{0} \phi .
$$

Substituting this result into Eq. (5) gives Eq. (22) for the flux, which verifies the continuum approach for this situation. 


\section{B. Linearized model: Structure evolution}

\section{Quiescent suspensions: Column formation}

Here we consider the evolution of the particle concentration resulting from the application of an electric field to a quiescent suspension $(\langle\mathbf{u}\rangle=\mathbf{0})$. Linearizing the conservation equation for small variations in concentration and for an applied field $\mathbf{E}=\left(0,0, E_{0}\right)$, Eq. (18) becomes

$$
\frac{\partial \phi^{\prime}}{\partial t}=-M\left(\frac{\partial^{2} \phi^{\prime}}{\partial x^{2}}+\frac{\partial^{2} \phi^{\prime}}{\partial y^{2}}-\kappa \frac{\partial^{2} \phi^{\prime}}{\partial z^{2}}\right)
$$

where $\phi^{\prime}(\mathbf{x}, t) \equiv \phi(\mathbf{x}, t)-\phi_{0}$ is the deviation of the volume fraction from its average value $\phi_{0}$,

$$
\begin{aligned}
M & =-\frac{a^{2} \epsilon_{0} E_{0}^{2} f\left(\phi_{0}\right)}{9 \eta_{c}}\left(\frac{\mathrm{d} \epsilon}{\mathrm{d} \phi}+\frac{\mathrm{d} a_{2}}{\mathrm{~d} \phi}\right)_{\phi_{0}}, \\
& =\frac{2 a^{2} \epsilon_{0} \epsilon_{c} \beta^{2} E_{0}^{2} f\left(\phi_{0}\right) \phi_{0}}{3 \eta_{c}\left(1-\beta \phi_{0}\right)^{3}},
\end{aligned}
$$

and $\kappa=2\left(1-\beta \phi_{0}\right) /\left(1+2 \beta \phi_{0}\right)>0$. Equation (24) is similar to a diffusion equation, with anisotropic diffusion and a negative diffusivity in the $x$ and $y$ directions. However, the particle phase motion has nothing to do with Brownian diffusion, but rather is caused by electrostatic forces. In fact, for typical ER suspensions, Brownian motion is negligible: for $a=1 \times 10^{-5} \mathrm{~m}, T=298 \mathrm{~K}$, and $\eta_{c}=0.1 \mathrm{Pas}$, the Brownian diffusivity is $D_{0}=k T / 6 \pi \eta_{c} a=2.2 \times 10^{-16} \mathrm{~m}^{2} / \mathrm{s}$, whereas $M=1.1 \times 10^{-9} \mathrm{~m}^{2} / \mathrm{s}$ for $\epsilon_{c}=2, \beta=1, E_{0}=1 \times 10^{6} \mathrm{~V} / \mathrm{m}$, and $\phi_{0}=0.1$.

The negative apparent diffusivity is reminiscent of spinodal decomposition in phase separating systems. ${ }^{21}$ Indeed, a uniform suspension in an electrostatic field is thermodynamically unstable. Consider the electrostatic contribution to the free energy, $\mathcal{F}^{E}=-\epsilon_{0} \epsilon(\phi) E^{2} / 2 .{ }^{22}$ Since $\partial^{2} \epsilon / \partial \phi^{2}>0$ [e.g., Eq. (7)], $\partial^{2} \mathcal{F}^{E} / \partial \phi^{2}<0$, which implies that the free energy of the suspension can be reduced by separating the system into dilute and concentrated phases.

We seek a solution of Eq. (24) of the form

$$
\phi^{\prime}(\mathbf{x}, t)=f(z) e^{i\left(k_{x} x+k_{y} y\right)} e^{s t},
$$

which represents fluctuations with sinusoidal variation in the $x$ and $y$ directions. The $z$ dependence, expressed through $f(z)$, is yet to be determined. The growth rate of the fluctuations is represented by $s$. Substitution of Eq. (27) into Eq. (24) yields

$$
\kappa f^{\prime \prime}(z)+\left(k_{x}^{2}+k_{y}^{2}-s / M\right) f(z)=0 .
$$

Using the no-flux boundary conditions $f^{\prime}(z)=0$ at $z=0$ and $z=L$ yields $f(z)=\cos n \pi z / L, n=0,1, \ldots$, and

$$
\frac{s L^{2}}{M}=\left(k_{x} L\right)^{2}+\left(k_{y} L\right)^{2}-\kappa n^{2} \pi^{2} .
$$

Fluctuations will grow for all $s>0$; the fastest growing fluctuations (for any given $k_{x}$ and $k_{y}$ ) will occur for $n=0$ and grow with rate $s_{\max } / M=k_{x}^{2}+k_{y}^{2}>0$. This result has several implications for the resulting suspension structures. First, since the fastest growing fluctuations occur for $n=0$, the resulting concentration profile should not depend on $z$. Sec- (a)

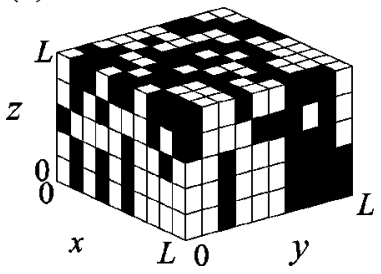

(b)

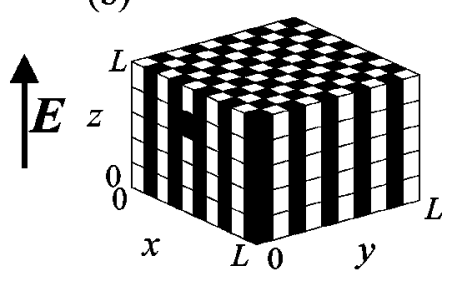

FIG. 1. (a) Initial structure and (b) structure after the application of an electric field to a quiescent suspension $\left(t M / L^{2}=0.05\right)$. Dark: positive concentration fluctuation. Light: negative concentration fluctuation.

ond, the maximum growth rate increases with $k_{x}$ and $k_{y}$; in fact, $s \rightarrow \infty$ as $k_{x}$ and $k_{y} \rightarrow \infty$. This implies that the fastest growing fluctuations will be thin structures. Third, the growth rate is symmetric with respect to $k_{x}$ and $k_{y}$, suggesting that if the initial fluctuations are random and isotropic, the resulting structures will be cylindrical columns:-i.e., columns of particles oriented in the $z$ direction, as commonly observed (a nonlinear analysis is required to determine the structures resulting from arbitrary initial conditions ${ }^{23}$ ).

This continuum analysis will break down at small length scales. The fastest growing wave numbers are certainly limited to at most roughly the inverse of the particle diameter. We also expect nonlocal polarization (i.e., "surface tension" ${ }^{24}$ ) to affect high $|\mathbf{k}|$ growth rates and thus the thickness of the resulting structures. However, these features will not alter the conclusion that columnar structures are produced. The effects of nonlocal polarization are revisited in Sec. II D.

A finite difference numerical solution of the governing equation [Eq. (24)] is illustrated in Fig. 1. No-flux boundary conditions were applied at the electrodes $\left(j_{z}=0\right.$ at $z$ $=0, L)$; periodic boundary conditions were applied at $x, y$ $=0, L$. The volume fraction was initially uniform $\left(\phi_{0}\right.$ $=0.1$ ) with small, random initial fluctuations. The evolution of the concentration fluctuations is illustrated in Fig. 1, where positive fluctuations are indicated by dark cubes and negative fluctuations are indicated by white cubes. The initial distribution and a distribution at a later time $\left(t M / L^{2}\right.$ $=0.05)$ are shown. As time progresses, the concentration fluctuations form columnar structures, which are similar to the fibrous aggregates commonly observed in ER and MR suspensions.

The mechanism of column formation is revealed by considering the change in electrostatic free energy associated with concentration fluctuations, given by $\delta \mathcal{F}^{E}=$ $-\epsilon_{0} \int_{V} \delta \epsilon(\phi) \mathbf{E} \cdot \mathbf{E}_{0} \mathrm{~d} V / 2,{ }^{22}$ where $\delta \epsilon$ is the fluctuation in dielectric constant caused by the concentration fluctuation, $\mathbf{E}$ is the electric field, and $\mathbf{E}_{0}$ is the electric field prior to the fluctuation. Consider first a parallel concentration fluctuation of the form $\phi(x)=\phi_{0}+A_{\|} \cos \beta_{\| x}$ with $A_{\|} \ll 1$. In this case the field is unaltered, and the change in the electrostatic free energy is $\delta \mathcal{F}^{E}=-\epsilon_{0} E_{0}^{2}\left(d^{2} \epsilon / d \phi^{2}\right) A_{\|}^{2} V / 8<0$, implying that such fluctuations are unstable and will continue to grow [using Eq. (7) for $\epsilon(\phi)]$. Next consider perpendicular fluctuations of the form $\phi(z)=\phi_{0}+A_{\perp} \cos \beta_{\perp} z$. In this case the field is altered by the fluctuation, and the resulting change in free energy is $\delta \mathcal{F}^{E}=+\epsilon_{0} E_{0}^{2}\left[(d \epsilon / d \phi)^{2} / \epsilon\right.$ 
$\left.-\left(d^{2} \epsilon / d \phi^{2}\right) / 2\right] A_{\perp}^{2} V / 4>0$, implying that such fluctuations are stable and will decay. Columnar fluctuations parallel to the applied field, which reduce the system free energy, will thus be favored.

\section{Sheared suspensions: Stripe formation}

Now we consider the evolution of the concentration profile when an electric field is applied to a uniform suspension under shear flow. For simple shear flow $\left[\langle\mathbf{u}\rangle=\left(\dot{\gamma}_{z}, 0,0\right)\right]$, the linearized conservation equation becomes

$$
\begin{aligned}
\frac{\partial \phi^{\prime}}{\partial t}+\dot{\gamma} z \frac{\partial \phi^{\prime}}{\partial x}= & -M\left(A_{1} \frac{\partial^{2} \phi^{\prime}}{\partial x^{2}}+A_{2} \frac{\partial^{2} \phi^{\prime}}{\partial y^{2}}-A_{3} \frac{\partial^{2} \phi^{\prime}}{\partial z^{2}}\right. \\
& \left.+A_{x z} \frac{\partial^{2} \phi^{\prime}}{\partial x \partial z}\right),
\end{aligned}
$$

where the dimensionless coefficients are

$$
\begin{aligned}
& A_{1}=1-\frac{2}{9} \frac{\dot{\gamma} L^{2}}{M}\left(\frac{a}{L}\right)^{2} f\left(\phi_{0}\right) \frac{\eta_{n}^{\prime}\left(\phi_{0}\right)}{\eta_{c}}, \\
& A_{2}=1-\lambda_{2} \frac{2}{9} \frac{\dot{\gamma} L^{2}}{M}\left(\frac{a}{L}\right)^{2} f\left(\phi_{0}\right) \frac{\eta_{n}^{\prime}\left(\phi_{0}\right)}{\eta_{c}}, \\
& A_{3}=\kappa+\lambda_{3} \frac{2}{9} \frac{\dot{\gamma} L^{2}}{M}\left(\frac{a}{L}\right)^{2} f\left(\phi_{0}\right) \frac{\eta_{n}^{\prime}\left(\phi_{0}\right)}{\eta_{c}}, \\
& A_{x z}=\frac{4}{9} \frac{\dot{\gamma} L^{2}}{M}\left(\frac{a}{L}\right)^{2} f\left(\phi_{0}\right) \frac{\eta_{p}^{\prime}\left(\phi_{0}\right)}{\eta_{c}},
\end{aligned}
$$

and where $\eta_{n}^{\prime}$ and $\eta_{p}^{\prime}$ denote $\mathrm{d} \eta_{n} / \mathrm{d} \phi$ and $\mathrm{d} \eta_{p} / \mathrm{d} \phi$, respectively. If the hydrodynamic contribution to the stress is ignored, then $A_{1}=A_{2}=1, A_{3}=\kappa$, and $A_{x z}=0$. We will examine the behavior with and without the hydrodynamic contribution.

We seek a solution of the form of Eq. (27), which upon substitution into Eq. (30) yields the ordinary differential equation for $f(z)$ :

$A_{3} f^{\prime \prime}(z)-i k_{x} A_{x z} f^{\prime}(z)+\left(A_{1} k_{x}^{2}+A_{2} k_{y}^{2}-s / M-i \dot{\gamma} k_{x} z / M\right) f$

$=0$.

Solution of this differential equation with boundary conditions $j_{z}=0$ at $z=0, L$ provides eigenvalues $s$ and eigenfunctions $f(z)$ for any values of the wave numbers $k_{x}$ and $k_{y}$. Stability is determined by the largest real part of the eigenvalue, designated by $s_{\max }\left(k_{x}, k_{y}\right)$.

Equation (35) was solved numerically to determine $s_{\max }\left(k_{x}, k_{y}\right)$. Consider first the evolution of the concentration fluctuations predicted when the hydrodynamic contribution to the stress is ignored (with $A_{1}=A_{2}=1, A_{3}=\kappa$, and $A_{x z}$ $=0)$. The results for $\dot{\gamma} L^{2} / M=10^{3}$ and $10^{4}$ are illustrated in Fig. 2, where contour plots of $s_{\max } L^{2} / M$ as a function of $k_{x}$ and $k_{y}$ are presented. The $s_{\max }=0$ contours in Figs. 2(a) and 2(b) represent the stability boundaries. The $s_{\max }=0$ contours are roughly semicircles, which grow with increasing shear rate. Thus certain fluctuations tend to be stabilized by shear flow.
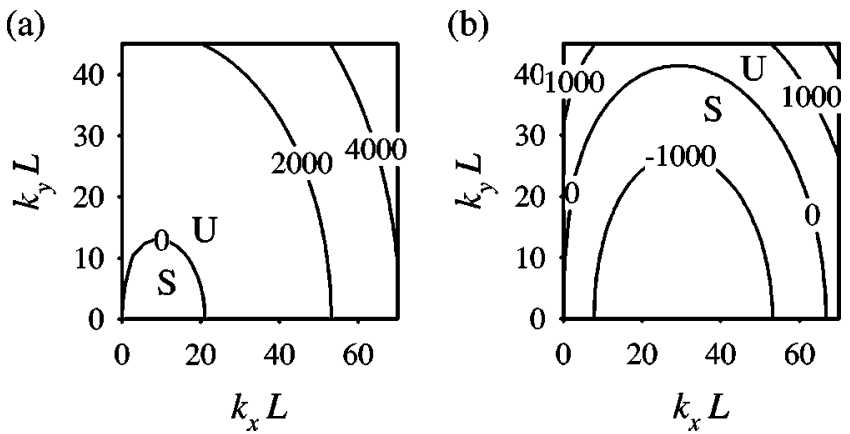

FIG. 2. Contour plots of $s_{\max } L^{2} / M$ as a function of $k_{x} L$ and $k_{y} L$ for (a) $\dot{\gamma} L^{2} / M=10^{3}$ and (b) $\dot{\gamma} L^{2} / M=10^{4}$. Stable and unstable regions are denoted by $\mathrm{S}$ and $\mathrm{U}$, respectively.

As discussed above the present model breaks down at large $|\mathbf{k}|$ because the physical dimensions of the particles and nonlocal polarization effects are neglected. Including these features will stabilize large $|\mathbf{k}|$ fluctuations, as discussed in Sec. II D. Combining this information with that presented in Fig. 2 implies that at sufficiently large shear rates, the only unstable fluctuations will be those with small $k_{x}$ and all $k_{y}$ below the large $|\mathbf{k}|$ cutoff (i.e., the region between the $s_{\max }$ $=0$ contour and the $k_{y}$ axis). Within this region, the most unstable fluctuations will be those with $k_{x}=0$ and nonzero $k_{y}$ - that is, the dominant structure is predicted to be stripes of particle-rich regions oriented in the flow direction, as commonly observed experimentally. ${ }^{7-11}$

Equation (30) has also been solved by the finite difference method to illustrate the stripe formation process. A no flux boundary condition was applied at the electrodes $\left(j_{z}\right.$ $=0$ at $z=0, L)$; periodic boundary conditions were applied at $x, y=0, L$. The evolution of concentration fluctuations is illustrated in Fig. 3 for $\dot{\gamma} L^{2} / M=10^{4}$ and $\phi_{0}=0.1$. The initially random fluctuations redistribute into stripes oriented in the flow direction, as described above. For the parameter values listed following Eq. (26) along with $L=10^{-3} \mathrm{~m}$, $\dot{\gamma} L^{2} / M=10^{4}$ corresponds to $\dot{\gamma}=10 \mathrm{~s}^{-1}$.

The mechanism of stripe formation in shear flow is related to the mechanism of column formation in quiescent suspensions. Fluctuations grow when parallel to the field and decay when perpendicular to the field. In shear flow, fluctuations parallel to the applied field will be rotated (and stretched) toward the flow direction, and thus will become (a)

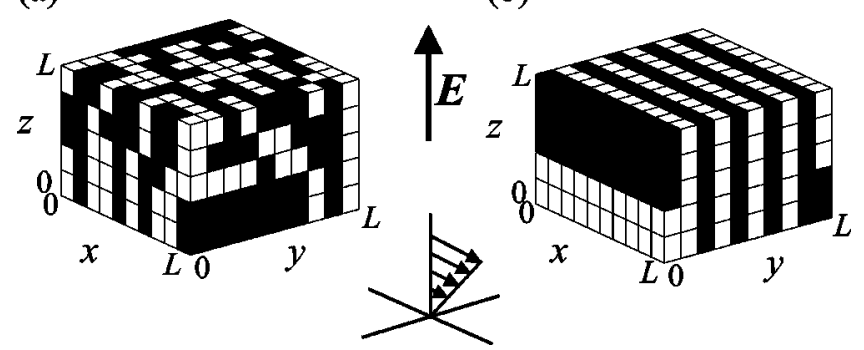

FIG. 3. (a) Initial structure and (b) structure after the application of an electric field to a flowing suspension $\left(\dot{\gamma} L^{2} / M=10^{4}, t M / L^{2}=0.05\right)$. Dark: positive concentration fluctuation. Light: negative concentration fluctuation. 


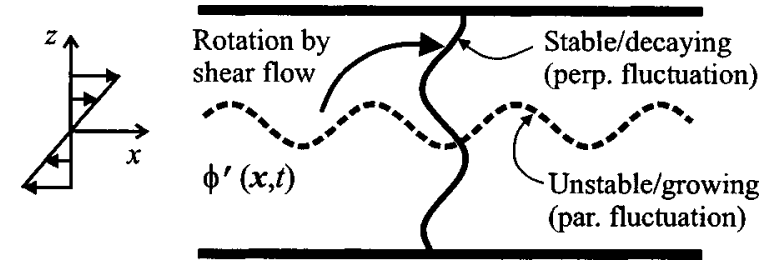

FIG. 4. Illustration of the stabilization of parallel fluctuations by simple shear flow.

stabilized, as illustrated in Fig. 4. The result is a uniform concentration in the plane of shear. However, since fluctuations in the vorticity direction [i.e., $\left.\mathbf{k}=\left(0, k_{y}, 0\right)\right]$ are unaffected by shear and will continue to grow, the resulting structure will be sheets of higher concentration in the plane of shear (i.e., stripes oriented in the flow direction).

Next consider the solution to Eq. (35) when the hydrodynamic contribution to the particle stress is included [i.e., when $A_{1}, A_{2}, A_{3}$, and $A_{x z}$ are given by Eqs. (31)-(34)]. A contour plot of the eigenvalues $s_{\max } L^{2} / M$ as a function of $k_{x}$ and $k_{y}$ for $\dot{\gamma} L^{2} / M=10^{4}$ is illustrated in Fig. 5(a). In Fig. 5(b), the contour plot without the hydrodynamic contribution to the stress is shown. The two contour plots are similar, suggesting that the hydrodynamic contribution to the stress does not affect the stability, at least for $\dot{\gamma} L^{2} / M=10^{4}$; the hydrodynamic contribution is even less important for smaller shear rates.

As the shear rate is increased further, one expects that the hydrodynamic contribution to the particle stress will eventually dominate over the electrostatic contribution and the uniform concentration profile will eventually become stable. In contrast, the maxima of the real parts of the eigenvalues in Eq. (35) become positive as $\dot{\gamma} \rightarrow \infty$, implying that the uniform profile is unstable even in the absence of electrostatic forces.

The appearance of the term $-M A_{x z} \partial^{2} \phi^{\prime} / \partial x \partial z$ in Eq. (24) leads to the counterintuitive prediction of instability of the strongly sheared suspension $\left(\dot{\gamma} L^{2} / M \rightarrow \infty\right)$ for certain fluctuations over a portion of the wave vector space satisfying $k_{x} k_{z}>0$ (wave vectors with a positive component along the axis of extension), at small to moderate $\phi$. It is shown in the Appendix that under the rheological modeling which has
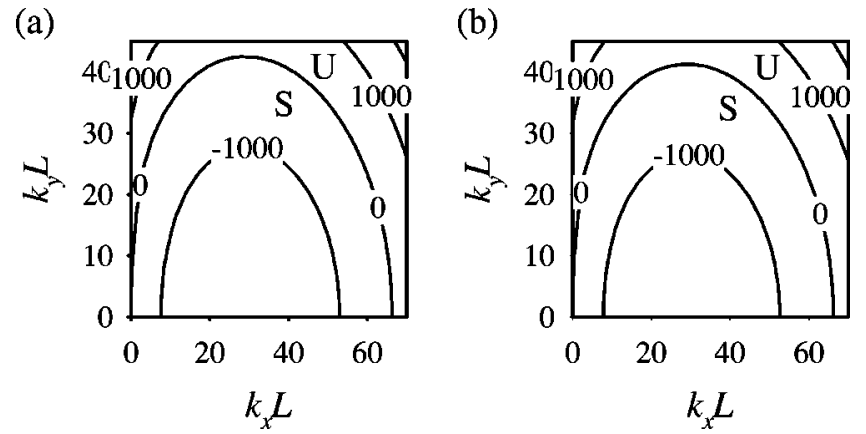

FIG. 5. Contour plots of $s_{\max } L^{2} / M$ as a function of $k_{x} L$ and $k_{y} L$ for $\dot{\gamma} L^{2} / M=10^{4}$ (a) with and (b) without the hydrodynamic contribution to the particle stress. Stable and unstable regions are denoted by $\mathrm{S}$ and $\mathrm{U}$, respectively.
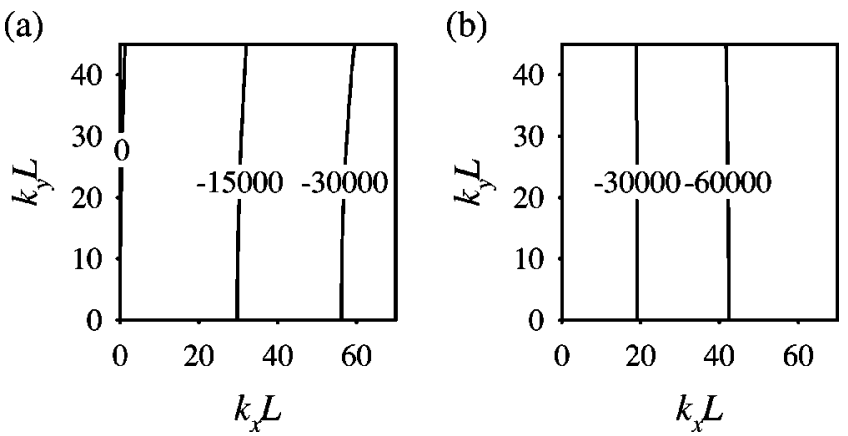

FIG. 6. Contour plots of $s_{\max } L^{2} / M$ as a function of $k_{x} L$ and $k_{y} L$ for $\phi_{0}$ $=0.1$ and $A_{x z}=0$, with (a) $\dot{\gamma} L^{2} / M=1 \times 10^{5}$ and (b) $\dot{\gamma} L^{2} / M=3 \times 10^{5}$.

provided accurate predictions of suspension flows in a range of geometries, ${ }^{16}$ this region is quite limited in extent at moderate $\phi$ and vanishes at elevated $\phi$. The eventual stability arises because compressive normal stresses serve as a stabilizing influence. The limited region of unstable wave vectors coupled with the rotational component of the flow means that a plane-wave fluctuation will reside in the zone of instability for only a limited period before being rotated into the stable zone. Furthermore, instability of a suspension of purely hydrodynamically interacting particles has not, to our knowledge, been reported in experiment or simulation.

The stability in the hydrodynamic limit is relevant to our examination of ER and MR effects with hydrodynamics. However, the predictions from theory and the lack of experimentally observed instability in the limit of a purely hydrodynamic suspension in shear flow lead us to neglect the $\partial^{2} \phi^{\prime} / \partial x \partial z$ term in Eq. (24) in the work to follow. This may be implemented by setting $A_{x z}=0$. Analysis and discussion of the predicted instability in the hydrodynamic limit is provided in the Appendix. For $A_{x z}=0$, the uniform concentration profile is stable in the absence of an electric field, regardless of the shear rate, as expected. For low shear rates, setting $A_{x z}=0$ only slightly modifies the stability boundary. For high shear rates, the system with $A_{x z}=0$ undergoes a transition in stability as illustrated in Fig. 6. For $\dot{\gamma} L^{2} / M=1$ $\times 10^{5}$, the system is unstable and stripes are produced. For $\dot{\gamma} L^{2} / M \geqslant 3 \times 10^{5}$, the uniform concentration profile is stable. The transition from stable to unstable is associated with a change in sign of the parameter $A_{2}$. This becomes more clear when we consider a one-dimensional version of the model in the following section.

\section{One-dimensional model: Steady-state structures}

In this section, we consider a simplified version of the model developed above, in which we assume that the particle concentration in shear flow is a function of only $y$ and $t$, where $y$ is the vorticity direction. This assumption is motivated by several factors. In experiments, shear flow appears to rapidly produce a concentration profile that is uniform in the flow $(x)$ and electric field $(z)$ directions. ${ }^{7-11}$ Analysis of the linearized model in the previous section also suggests that the suspension evolves to a structure that only varies in the vorticity $(y)$ direction. Finally, a full description of structure evolution with arbitrary, finite amplitude fluctuations re- 


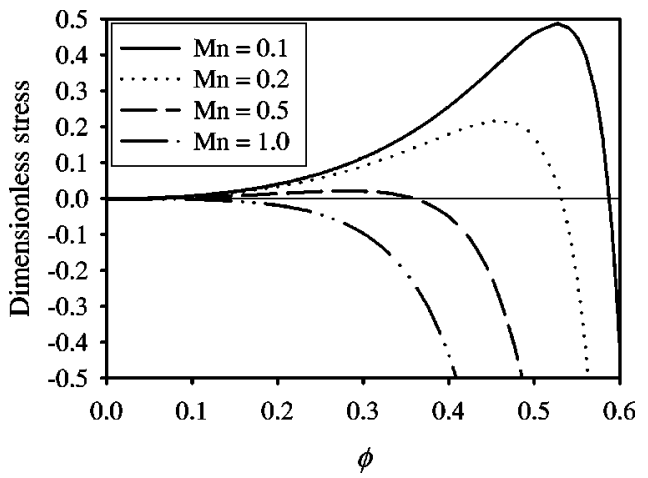

FIG. 7. Dimensionless normal stress, $\sigma_{y y}^{p} / 2 \epsilon_{0} \epsilon_{c} \beta^{2} E^{2}$, as a function of $\phi$ for different Mn.

quires the simultaneous solution of four nonlinear partial differential equations: the particle conservation equation, the bulk suspension momentum balance, the bulk suspension mass balance, and Gauss' law. The assumption $\phi=\phi(y, t)$ removes the coupling between these equations, permitting the analysis of only the particle conservation equation (i.e., this assumption maintains simple shear flow and a uniform electric field in the $z$ direction).

Assuming $\phi=\phi(y, t)$, the particle conservation equation (18) becomes

$$
\frac{\partial \phi}{\partial t}=-\frac{2 a^{2}}{9 \eta_{c}} \frac{\partial}{\partial y}\left(f(\phi) \frac{\partial \sigma_{y y}^{p}}{\partial y}\right),
$$

where $\sigma_{y y}^{p}$ is the electrostatic plus hydrodynamic contribution to the normal particle stress in the vorticity direction, which can be expressed

$$
\sigma_{y y}^{p}=2 \epsilon_{0} \epsilon_{c} \beta^{2} E^{2} \phi^{2}\left[\frac{3}{4(1-\beta \phi)^{2}}-\frac{\lambda_{2} K_{n} \mathrm{Mn}}{\left(\phi_{\max }-\phi\right)^{2}}\right],
$$

where

$$
\mathrm{Mn}=\frac{\eta_{c} \dot{\gamma}}{2 \epsilon_{0} \epsilon_{c} \beta^{2} E^{2}}
$$

is the Mason number.

In Fig. 7, $\sigma_{y y}^{p}$ is plotted as a function of $\phi$ for different Mn (using $\beta=1, \lambda_{2}=0.5, K_{n}=0.75$, and $\phi_{\max }=0.68$ ). For fixed $\dot{\gamma}$ and $\mathbf{E}, \sigma_{y y}^{p}$ can be treated as an explicit function of $\phi(y, t)$ only. The flux can then be expressed $\quad j_{y}=\left[2 a^{2} f(\phi) / 9 \eta_{c}\right]\left(d \sigma_{y y}^{p} / d \phi\right)(\partial \phi / \partial y)$, where $\left[2 a^{2} f(\phi) / 9 \eta_{c}\right]\left(d \sigma_{y y}^{p} / d \phi\right)$ represents a gradient diffusivity. ${ }^{25,26}$ Thus the $\phi$ dependence of $\sigma_{y y}^{p}$ illustrated in Fig. 7 provides information about the system behavior. For vanishing $\phi, d \sigma_{y y}^{p} / d \phi \rightarrow 0$, and hence the particle flux vanishes. For larger $\phi$, the behavior depends on $\mathrm{Mn}$.

For large $\mathrm{Mn}($ e.g., $\mathrm{Mn}>1), d \sigma_{y y}^{p} / d \phi \leqslant 0$ for all concentrations. Thus the particle flux is always directed opposite to the concentration gradient (i.e., like "normal" Fickian diffusion). The steady state in this case is thus a uniform concentration profile. For this range of $\mathrm{Mn}$, the hydrodynamic contribution to the particle stress always dominates over the electrostatic stress, independent of $\phi$.
For small Mn, however, the variation of the stress with $\phi$ can be negative or positive. For small $\phi$ where $d \sigma_{y y}^{p} / d \phi$ $>0$, the particle flux is in the same direction as the concentration gradient. Hence concentration gradients are amplified, analogous to phase separation via spinodal decomposition. ${ }^{21}$ This occurs because the electrostatic contribution to the particle stress dominates over the hydrodynamic contribution, resulting in a tendency to increase locally the particle concentration. The particle accumulation continues until the suspension only consists of regions without particles and regions with sufficiently large concentration such that $\partial j_{y} / \partial y$ $=0$ (discussed in more detail below). Thus for small Mn, there is a nonuniform steady state consisting of particle-free regions and regions of high concentration. This "phase separation" will only occur when the initial concentration profile consists of regions of sufficiently low concentration where $d \sigma_{y y}^{p} / d \phi>0$.

In the remainder of this section, we consider the structure evolution predicted by this one-dimensional model. We consider first a linear stability analysis, where we find that the critical Mason number for stripe formation is identical to that predicted from the three-dimensional model. We then analyze the steady-state structure predicted. Below the critical Mason number, the concentration within the stripes increases as $\mathrm{Mn}$ is decreased.

\section{Linear stability analysis}

With the assumption $\phi=\phi(y, t)$, the linearized particle conservation equation (30) reduces to

$$
\frac{\partial \phi^{\prime}}{\partial t}=-M A_{2} \frac{\partial^{2} \phi^{\prime}}{\partial y^{2}},
$$

where $\phi^{\prime}=\phi-\phi_{0}$ and $M$ is given by Eq. (26). The quantity $A_{2}$ can be written in terms of the Mason number:

$$
A_{2}=1-\frac{2 \lambda_{2} \eta_{n}^{\prime}\left(\phi_{0}\right)\left(1-\beta \phi_{0}\right)^{3}}{3 \eta_{c} \phi_{0}} \mathrm{Mn} \text {. }
$$

Seeking a solution of the form $\phi^{\prime}=e^{i k_{y} y} e^{s t}$, the eigenvalues are

$$
\frac{s L^{2}}{M}=A_{2}\left(k_{y} L\right)^{2} .
$$

Thus the uniform profile is stable if $A_{2}<0$. This is identical to the result obtained with the three-dimensional model (with $A_{x z}=0$ ) in Sec. II B 2, providing some assurance that the one-dimensional model retains the correct physical origin of stripe formation.

The stability boundary $\left(A_{2}=0\right)$ can be characterized by a critical Mason number

$$
\mathrm{Mn}_{c}=\frac{3 \phi_{\max }^{2}}{4 \lambda_{2} K_{n}} \frac{\left(1-\phi_{0} / \phi_{\max }\right)^{3}}{\left(1-\beta \phi_{0}\right)^{3}} .
$$

The critical Mason number is plotted as a function of $\phi_{0}$ in Fig. 8. For $\mathrm{Mn}>\mathrm{Mn}_{c}$, all eigenvalues are negative and the uniform concentration profile is stable. For $\mathrm{Mn}<\mathrm{Mn}_{c}$, the uniform concentration profile is unstable and a "phase separation" will begin. Since the wave number $k_{y} \rightarrow \infty$ is the most unstable, this linearized model predicts that infinitely thin 


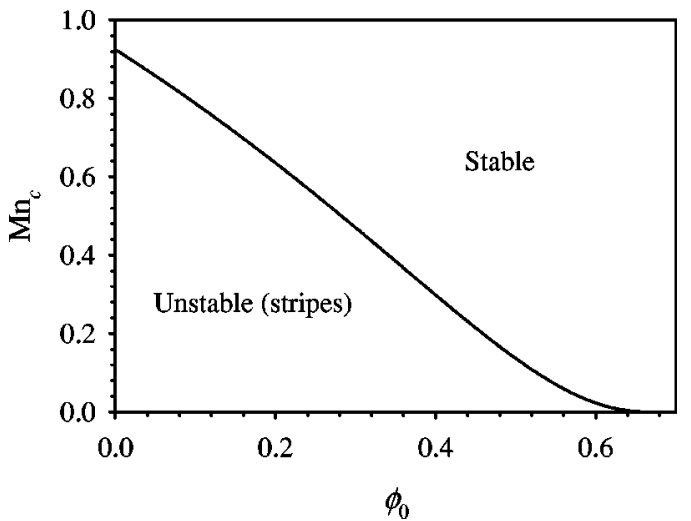

FIG. 8. Critical Mason number as a function of $\phi_{0}$.

stripes will begin to form most rapidly. To understand the later stages of structure evolution, the full nonlinear model must be analyzed. The steady states predicted by the nonlinear model are considered below. Nonlocal contributions, considered in Sec. II D, do not alter the stability boundary, but produce a finite value for the fastest growing wave number.

\section{Steady-state structures}

Here we consider the steady-state structures predicted by the one-dimensional model. At steady state, Eq. (36) reduces to

$$
0=-\frac{d j_{y}}{d y}=-\frac{2 a^{2}}{9 \eta_{c}} \frac{d}{d y}\left(f(\phi) \frac{d \sigma_{y y}^{p}}{d y}\right),
$$

and hence the particle flux $j_{y}$ must be constant. Assuming either a finite domain with no-flux boundary conditions or an infinite domain with uniform concentration at infinity, $j_{y}$ $=0$ everywhere at steady state. This implies that $f(\phi)$ $\times\left(d \sigma_{y y}^{p} / d y\right)=0$; since $f(\phi)>0$, this implies that $\sigma_{y y}^{p}$ $=$ constant at steady state.

Consider the transient structure evolution, under conditions where the uniform steady state is unstable (Mn $\left.<\mathrm{Mn}_{c}\right)$. As described at the beginning of this section, regions of small $\phi$ give $d \sigma_{y y}^{p} / d \phi>0$, producing a flux in the direction of the concentration gradient. This "uphill" flux will tend to reduce the concentration in regions of small $\phi$. Since $d \sigma_{y y}^{p} / d \phi$ will remain positive as $\phi \rightarrow 0$, the small $\phi$ regions will continue to decrease in concentration until $\phi$ $=0$. At $\phi=0, \sigma_{y y}^{p}=0$, and thus, along with the analysis in the previous paragraph, the nonuniform steady-state concentration profile must satisfy

$$
\sigma_{y y}^{p}=0
$$

throughout the suspension. This implies that the steady state is one in which the opposing electrostatic (tensile) and hydrodynamic (compressive) contributions to $\sigma_{y y}^{p}$ balance.

Substituting Eq. (37) for $\sigma_{y y}^{p}$ into Eq. (44) yields

$$
\phi^{2}\left(\frac{3}{4(1-\beta \phi)^{2}}-\frac{\lambda_{2} K_{n} \mathrm{Mn}}{\left(\phi_{\max }-\phi\right)^{2}}\right)=0 .
$$

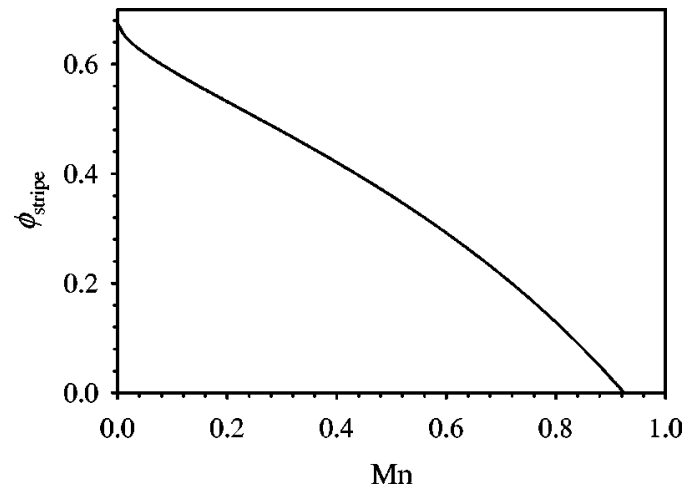

FIG. 9. Stripe concentration as a function of $\mathrm{Mn}$.

For $\mathrm{Mn}<3 \phi_{\max }^{2} /\left(4 \lambda_{2} K_{n}\right)$, this equation has two physical solutions, $\phi=0$ and $\phi=\phi_{\text {stripe }}$, where

$\phi_{\text {stripe }}=\frac{4 \beta \lambda_{2} K_{n} \mathrm{Mn}-3 \phi_{\max }+2 \sqrt{3\left(1-\beta \phi_{\max }\right)^{2} \lambda_{2} K_{n} \mathrm{Mn}}}{4 \beta^{2} \lambda_{2} K_{n} \mathrm{Mn}-3}$.

Hence, for sufficiently small Mn, this model predicts a nonuniform steady-state concentration profile with regions with $\phi=0$ and regions with $\phi=\phi_{\text {stripe }}$. Since we have assumed that $\phi$ depends only on $y$, this steady state represents stripes oriented in the flow direction.

In Fig. $9, \phi_{\text {stripe }}$ is plotted as a function of Mn. The stripe concentration does not depend on the average concentration $\phi_{0}$. However, there will obviously only be a nonuniform steady state if $\phi_{\text {stripe }}>\phi_{0}$, or in terms of the Mason number, if $\operatorname{Mn}<3\left(\phi_{\max }-\phi_{0}\right)^{2} /\left[4 \lambda_{2} K_{n}\left(1-\beta \phi_{0}\right)^{2}\right]$. The stripe concentration increases with decreasing Mn, approaching $\phi_{\max }$ as $\mathrm{Mn} \rightarrow 0$. This trend agrees with experiments reported by Viera et al.,${ }^{11}$ where the thickness and number of stripes increased with increasing shear rate, implying that the concentration within the stripes decreased with increasing Mn.

This analysis does not provide predictions about the number or thickness of the stripes. These will be influenced by nonlocal polarization, which will create a "surface tension" 24 at discontinuities in $\phi(y)$. Minimization of the total free energy implies that the steady state should consist of a single stripe with only two interfaces. Analysis of the transient approach to this steady state requires analysis of the nonlinear, unsteady-state conservation equation, which is beyond the scope of this study.

Combining the results of the linear stability analysis with that presented above for the nonuniform steady-state behavior, a complete description of the steady-state behavior of ER suspensions can be constructed. This is illustrated in Fig. 10 where $\max \phi$ is plotted as a function of Mn (for $\phi_{0}=0.3$ ). Here, $\max \phi$ is defined as the maximum concentration in the suspension. At large $\mathrm{Mn}>\mathrm{Mn}_{c}$, the suspension is uniform and $\max \phi=\phi_{0}$. Decreasing Mn initially does not alter the steady-state profile, but moves the system closer to the stability boundary. At $\mathrm{Mn}=\mathrm{Mn}_{c}$, the uniform steady state becomes unstable and the system transforms into a nonuniform concentration profile with $\max \phi=\phi_{\text {stripe }}\left(\mathrm{Mn}_{c}\right)$. Decreasing Mn further increases the concentration within the stripes, with $\phi_{\text {stripe }}$ given by Eq. (46). When Mn is then increased, 


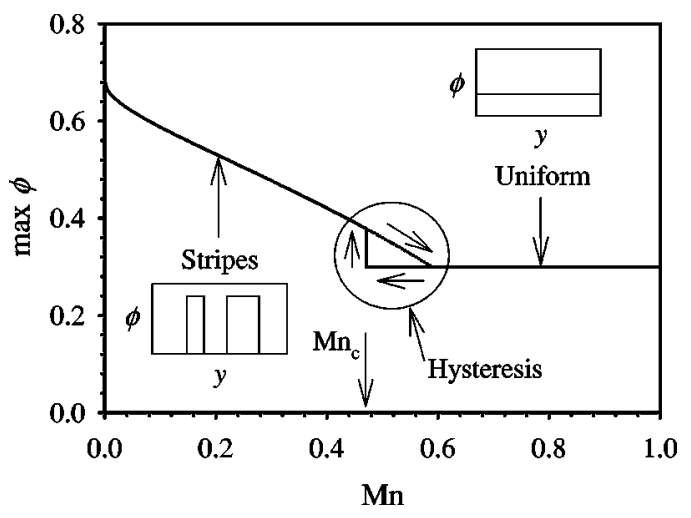

FIG. 10. Maximum concentration in a suspension as a function of $\mathrm{Mn}$ for $\phi_{0}=0.3$.

the concentration within the stripes decreases. When $\mathrm{Mn}$ is increased just above $\mathrm{Mn}_{c}$, the nonuniform concentration is still a valid solution of Eq. (43), with $\phi_{\text {stripe }}>\phi_{0}$. Assuming that the nonuniform profile remains stable, the stripe structure will persist until $\mathrm{Mn}$ is increased to the point where $\phi_{\text {stripe }}=\phi_{0}$, i.e., where the total thickness of the stripe(s) occupies the entire suspension volume. At this point, the structure is uniform and stable, and thus subsequent small increases or decreases in $\mathrm{Mn}$ should maintain a uniform structure.

The one-dimensional model thus appears to predict hysteretic behavior for the steady-state concentration profile. The hysteresis is predicated on the assumption of two stable steady-state solutions over a certain range of Mn. As described previously, the stability of the nonuniform profile will be influenced by nonlocal polarization, which should be included before the hysteresis is explored further. The predicted hysteresis occurs over a small range of $\mathrm{Mn}$ and thus is probably of little importance in application of ER and MR fluids.

As $\phi_{0}$ is varied, $\mathrm{Mn}_{c}$ is altered but the dependence of $\phi_{\text {stripe }}$ on $\mathrm{Mn}$ is unaffected. The dependence of the structure on Mn and $\phi_{0}$ can thus be represented by a series of curves illustrated in Fig. 11 (which again is a combination of information obtained from the linear stability and the steady-state analyses). At sufficiently large $\mathrm{Mn}$, all suspensions remain uniform. At $\mathrm{Mn}_{c}$, which depends on $\phi_{0}$, the structure discontinuously changes to a nonuniform striped structure with $\phi_{\text {stripe }}>\phi_{0}\left[\mathrm{Mn}_{c}\left(\phi_{0}\right)\right.$ is represented by the dashed curve in Fig. 11]. Once in the nonuniform state, the systems move along a common curve $\phi_{\text {stripe }}(\mathrm{Mn})$, independent of $\phi_{0}$.

\section{Effects of nonlocal polarization}

In this section, we briefly examine the effects of a model of nonlocal polarization in the linear stability analyses presented above. This analysis will be presented in more detail in a future publication. ${ }^{28}$

$\mathrm{Cahn}^{21}$ examined the free energy and structure evolution in phase-separating, binary solutions of species $A$ and $B$. In regions where the composition is nonuniform, the free energy density depends on the local composition, as well as the composition in the surrounding environment. For small

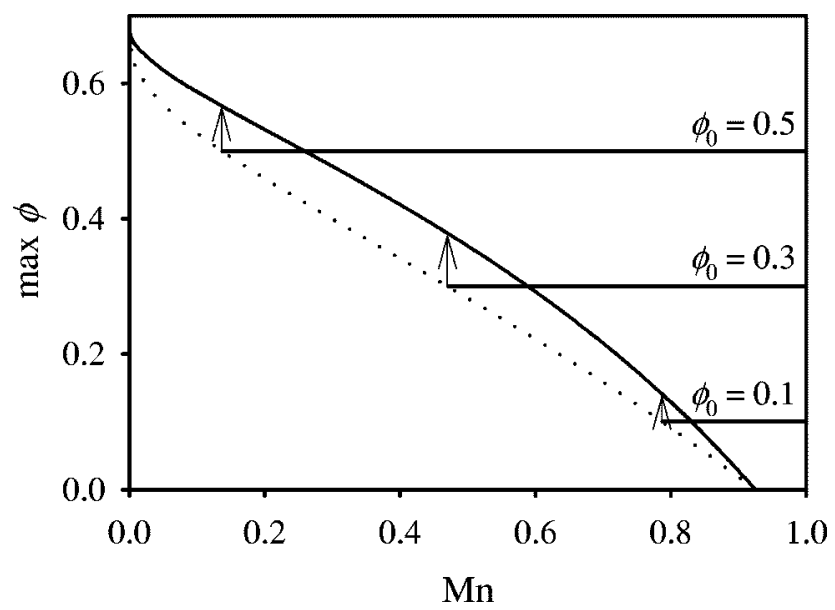

FIG. 11. Maximum concentration in a suspension as a function of Mn for different $\phi_{0}$.

variations in composition, the influence of the surrounding environment can be expressed in terms of derivatives in concentration. For isotropic systems, the free energy density is thus $^{27}$

$f\left(c, \nabla c, \nabla^{2} c, \ldots\right)=f_{0}(c)+\kappa_{1} \nabla^{2} c+\kappa_{2}(\nabla c)^{2}+\cdots$,

where $f_{0}(c)$ is the free energy density of a uniform system at concentration $c, c$ is the mole fraction of species $B$, and $\kappa_{1}$ and $\kappa_{2}$ are phenomenological coefficients. Proposing that the flux of species $A$ can be expressed $\mathbf{j}_{A}=-\mathcal{M} \nabla\left(\mu_{A}-\mu_{B}\right)$, where $\mathcal{M}$ is a positive mobility and $\mu_{A}$ and $\mu_{B}$ are the chemical potentials of species $A$ and $B$, respectively, Cahn ${ }^{21}$ derived a conservation equation to describe the initial stages of the evolution of the concentration profile,

$$
\frac{\partial c}{\partial t}=\mathcal{M}\left(\frac{\partial^{2} f_{0}}{\partial c^{2}}\right) \nabla^{2} c-2 \mathcal{M} K \nabla^{4} c,
$$

where $K \equiv-d \kappa_{1} / d c+\kappa_{2}>0$. Thus the nonlocal contribution to the free energy gives rise to a $\nabla^{4}$ term in the linearized conservation equation.

In ER suspensions, the free energy should also contain nonlocal contributions owing to the nonlocal character of the particle polarization. The polarization of a particle is proportional to the local electric field, which in turn is the sum of the applied field plus the disturbance fields generated by all of the other polarized particles. Thus we expect the particle polarization, and thus the electrostatic contribution to the free energy density, to depend on the local concentration as well as the concentration distribution in the neighborhood of the point of interest. Following $\mathrm{Cahn}^{21}$ we therefore expect a modified linearized particle conservation equation of the form

$$
\begin{aligned}
\frac{\partial \phi^{\prime}}{\partial t}+\dot{\gamma} z \frac{\partial \phi^{\prime}}{\partial x}= & -M\left(A_{1} \frac{\partial^{2} \phi^{\prime}}{\partial x^{2}}+A_{2} \frac{\partial^{2} \phi^{\prime}}{\partial y^{2}}-A_{3} \frac{\partial^{2} \phi^{\prime}}{\partial z^{2}}\right. \\
& \left.+A_{x z} \frac{\partial^{2} \phi^{\prime}}{\partial x \partial z}\right)-C M \nabla_{2}^{4} \phi^{\prime}
\end{aligned}
$$


where $C$ is a positive coefficient, $M$ is the mobility parameter introduced in Sec. II B 1 , and $\nabla_{2}^{4} \equiv \partial^{4} / \partial x^{4}+\partial^{4} / \partial y^{4}$. We have ignored the nonlocal contribution in the $z$ direction, since the nonlocal contributions tend to stabilize fluctuations and the fastest growing wave forms are uniform in the $z$ direction.

The addition of the $\nabla^{4}$ term to the particle conservation equation has a modest impact on the linear stability analysis. In quiescent suspensions, cylindrical columns are still produced for random, isotropic initial fluctuations. ${ }^{29}$ However, the fastest growing wave numbers are no longer infinite, but given by $k_{x}=k_{y}=1 / \sqrt{2 C}$. Thus the thickness of columnar structures produced initially should have a diameter $d$ $\approx \pi \sqrt{2 C}$. A self-consistent dipole analysis gives $C \propto a^{2},{ }^{28}$ and thus the columns are still expected to be thin initially.

For ER suspensions under shear, consider the onedimensional model $\phi=\phi(y, t)$. The presence of the $\nabla_{2}^{4}$ term in Eq. (49) yields the eigenvalues ${ }^{29}$

$$
\frac{s L^{2}}{M}=\left(A_{2}-C k_{y}^{2}\right) k_{y}^{2} L^{2} .
$$

The critical Mason number describing the stability boundary $(s=0)$ is unaltered by the $\nabla_{2}^{4}$ term, but the fastest growing wave numbers are reduced from $k_{y} \rightarrow \infty$ to $k_{y}$ $=\sqrt{\left|A_{2}\right| /(2 C)}$, implying the formation of stripes of small but nonzero thickness. The maximum growth rate is now finite; using $C=a^{2}$, the maximum growth rate is $s_{\max }$ $=\left(M / 4 a^{2}\right)\left(1-\mathrm{Mn} / \mathrm{Mn}_{c}\right)^{2}$, where $\mathrm{Mn}_{c}$ is given by Eq. (42). Using the parameter values employed in Sec. II B 1 , the growth rate in the limit of small $\mathrm{Mn}$ is $s_{\max }=2.7 \mathrm{~s}^{-1}$. This prediction is consistent with the appearance of stripes on experimental time scales.

Nonlocal effects will also influence the nonuniform steady-state structures obtained, but will not alter the result that stripes are indeed produced. Analysis of nonlocal contributions to this full nonlinear model will be the subject of a future publication.

\section{CONCLUSION}

A two-fluid continuum model for mass transport in ER suspensions has been presented. The particle flux is related to the divergence of the particle contribution to the stress, which in turn is related to the suspension dielectric and electrostrictive properties. Solutions of the resulting particle conservation equation capture common observations: column formation in quiescent suspensions and stripe formation in sheared suspensions. Columns form because only these structures produce a decrease in the free energy. Stripes form in shear flow because the flow stabilizes fluctuations in the plane of shear.

Although this model successfully reproduces several aspects of ER and MR suspension behavior, important features are missing that should be included in the future. Nonlocal contributions to the stress and flux should be included to correctly capture the large- $|\mathbf{k}|$ behavior, as well as the later stages of structure evolution and the details of interfaces at steady state. Although the isotropic model developed here produces a purely viscous field-induced increase in the apparent suspension viscosity, ${ }^{29}$ the influence of structural an- isotropy on the electrostatic particle contribution to the stress must be included in order to capture the dramatic fieldinduced rheological phenomena commonly observed (e.g., field-dependent storage moduli and yield stresses). ${ }^{30}$ Finally, nonlinear magnetization must also be addressed in order to probe MR suspensions under large magnetic field strengths, as commonly found in practical applications.

\section{APPENDIX}

In the absence of ER or MR effects or presumably, for very large $\dot{\gamma} L^{2} / M$, the stability of the suspension flow is determined by hydrodynamics. We assume that the suspension is noncolloidal with negligible interparticle forces except at, or very near, contact so that the suspension rheology proposed in Morris and Boulay ${ }^{16}$ is applicable. Instability of simple shear of such suspensions has not, to our knowledge, been reported. Here we examine the linear stability predictions based upon the suspension flow model employed in this work.

Prior development leads to the equation for particle mass conservation, which for purely hydrodynamic conditions is given by

$$
\frac{\partial \phi}{\partial t}+\mathbf{u} \cdot \nabla \phi=-\frac{2 a^{2}}{9 \eta_{c}} \nabla \cdot\left[f(\phi) \nabla \cdot \boldsymbol{\sigma}^{(p, H)}\right],
$$

where $\mathbf{u}$ is the suspension averaged velocity.

In the form proposed by Morris and Boulay ${ }^{16}$ for shear flows, the hydrodynamic stress contribution of the particles, $\boldsymbol{\sigma}^{(p)}$, is given by

$$
\boldsymbol{\sigma}^{(p, H)}=-\eta_{n}(\phi) \dot{\gamma} \hat{\mathbf{Q}}+2 \eta_{p} \mathbf{e},
$$

where $\dot{\gamma} \equiv \sqrt{2 \mathbf{e}: \mathbf{e}}$ with $\mathbf{e}$ the local strain rate of the bulk suspension. The tensor $\hat{\mathbf{Q}}$ is in general nonisotropic, but diagonal and having positive entries, in a simple-shear flow; modeling for other flows has not been proposed.

It is assumed that a base state of uniform particle concentration $\phi_{0}$ and uniform shear rate $\dot{\gamma}=\left(\partial u_{x} / \partial z\right)_{0}$ is perturbed through fluctuation in the particle fraction, $\phi=\phi_{0}$ $+\phi^{\prime}$. Because the base state satisfies $\nabla \cdot \sigma^{(p, H)}=0$, the variation of $f$ has effect only at quadratic and higher level in $\phi^{\prime}$; for similar reasons, the flow may be taken as a simple shear in the left side of the equation. The equation for $\phi^{\prime}$ may thus be written

$$
\frac{\partial \phi^{\prime}}{\partial t}+\dot{\gamma} z \frac{\partial \phi^{\prime}}{\partial x}=-\frac{2 a^{2} f(\phi)}{9 \eta_{c}} \nabla \cdot\left(\nabla \cdot \boldsymbol{\sigma}^{(p, H)}\right) .
$$

As noted in the main text, the mixed derivative term $-M A_{x z} \partial^{2} \phi^{\prime} / \partial x \partial z$ appearing in Eq. (24) leads to a predicted instability at large shear rate. ${ }^{29}$ The mixed derivative results from the shear stress portion of $\nabla \cdot \boldsymbol{\sigma}^{(p, H)}$; while the flow may vary from simple shear due to variations in $\phi$, this effect does not appear at linear order in $\phi^{\prime}$ in the mixed derivative term. Nor can this term in general be balanced by normal stress contributions owing to the difference in the scaling with $\phi_{0}$. Hence, the flow is considered to remain 
simple shear in the linear stability analysis, and this does not appear to affect the generality of the conclusions. We thus consider the equation

$$
\begin{aligned}
\frac{\partial \phi^{\prime}}{\partial t}+\dot{\gamma} z \frac{\partial \phi^{\prime}}{\partial x} & =-\frac{2 a^{2}}{9 \eta_{c}} f \dot{\gamma} \frac{\partial}{\partial x_{i}} \frac{\partial}{\partial x_{j}}\left(-\eta_{n} \hat{Q}_{i j}+\eta_{p} \hat{e}_{i j}\right) \\
& =-\frac{2 a^{2}}{9 \eta_{c}} f \dot{\gamma}\left(-\eta_{n}^{\prime} \hat{Q}_{i j}+\eta_{p}^{\prime} \hat{e}_{i j}\right) \frac{\partial^{2} \phi^{\prime}}{\partial x_{i} \partial x_{j}},
\end{aligned}
$$

in which $\hat{\mathbf{e}}=2 \mathbf{e} / \dot{\gamma}$. It is convenient to use the "natural" coordinates of the stress, in which only normal stress terms appear. ${ }^{31}$ For this purpose, it is simplest to take the normal stress tensor to be isotropic, a slight (and apparently inconsequential for present purposes) variation from the system with normal stress differences as modeled in Morris and Boulay. ${ }^{16}$ The coordinate directions natural to the particle contribution to the stress then lie along the lines $z=x$ (direction of extension) and $z=-x$ (direction of compression), with the neutral direction along $y$; i.e., the natural directions are along the eigenvectors of $\mathbf{e}$ and are rotated by $\pi / 4$ radians counterclockwise in the plane from the original triad. The particle stress in this Cartesian frame is now given by

$$
\boldsymbol{\sigma}^{(p, H)}=\dot{\gamma}\left(\begin{array}{ccc}
-\eta_{n}+\eta_{p} & 0 & 0 \\
0 & -\eta_{n} & 0 \\
0 & 0 & -\left(\eta_{n}+\eta_{p}\right)
\end{array}\right),
$$

with the direction of extension now labeled 1 (and having entry $-\eta_{n}+\eta_{p}$ in upper left), the direction of compression 3 , and the neutral direction 2. Introducing this form for $\boldsymbol{\sigma}^{p}$ to Eq. (A5) and using the standard disturbance form for linear analysis of $\phi^{\prime}=e^{i\left(k_{1} x_{1}+k_{2} x_{2}+k_{3} x_{3}\right)+s t}$, we obtain

$$
\begin{aligned}
s+\frac{\sqrt{2}}{2} i\left(k_{3} x_{1}+k_{1} x_{3}\right)= & -\frac{2 a^{2}}{9 \eta_{c}} f \dot{\gamma}\left[-k_{1}^{2}\left(-\eta_{n}^{\prime}+\eta_{p}^{\prime}\right)\right. \\
& \left.-k_{2}^{2} \eta_{n}^{\prime}+k_{3}^{2}\left(\eta_{n}^{\prime}+\eta_{p}^{\prime}\right),\right] .
\end{aligned}
$$

The condition for instability $[\operatorname{Re}(s)>0]$ is

$$
k_{1}^{2}\left(-\eta_{p}^{\prime}+\eta_{n}^{\prime}\right)-k_{2}^{2} \eta_{n}^{\prime}-k_{3}^{2}\left(\eta_{n}^{\prime}+\eta_{p}^{\prime}\right)>0 .
$$

Since fluctuations in the neutral direction $\left(x_{2}\right)$ are predicted to be stabilized by hydrodynamic normal stresses, the most unstable modes are those in the plane of shear and we set $k_{2}=0$ to find the region of unstable wave vectors:

$$
\left|\frac{k_{1}}{k_{3}}\right|>\sqrt{\frac{\eta_{n}^{\prime}+\eta_{p}^{\prime}}{\eta_{p}^{\prime}-\eta_{n}^{\prime}}}, \text { and } \eta_{p}^{\prime}>\eta_{n}^{\prime} .
$$

For small $\phi$, the latter condition is met, as $\eta_{p}$ is linear in $\phi$ whereas $\eta_{n}$ goes as $\phi^{2}$. Thus, for small bulk particle fraction where normal stresses are negligible, fluctuations in the shear plane are predicted to be unstable for $\left|k_{1}\right|>\left|k_{3}\right|$-i.e. for all wave vectors having a positive scalar product with the extensional eigenvector.

Compressive normal stresses, here modeled as isotropic and thus equivalent to a dispersive "pressure," have a stabilizing influence and thus reduce the extent of the unstable zone. With the modeling of Morris and Boulay ${ }^{16}$ for $\eta_{n}$ and $\eta_{p}$, the extent of the zone of instability decreases from that satisfying $\left|k_{1}\right|>\left|k_{3}\right|$ at $\phi_{0}=0$ to $\left|k_{1}\right|>4\left|k_{3}\right|$ at about $\phi_{0}$ $=0.4 \phi_{\max }$. The progressively narrower "wedge" of unstable wave vectors vanishes when $\eta_{n}^{\prime}>\eta_{p}^{\prime}$ for $\phi_{0}>0.6 \phi_{\max }$ and no longer satisfies the second condition of Eq. (A9).

In this work, we have neglected the influence of this instability. The basis for the decision has two parts. First, the predicted instability is limited to relatively small $\phi$ under the normal stress model employed and the region of unstable wave vectors is found to be quite limited in extent at practical values of $\phi_{0}$. Because the flow has a rotational component, any fluctuation within the unstable zone will reside there only for times of $O\left(\dot{\gamma}^{-1}\right)$, with this time quickly reduced with increasing $\phi_{0}$ as the unstable zone contracts. Second, in neither experiments nor in simulations has evidence of the purely hydrodynamic instability of the particle fraction of a near hard-sphere suspension been reported, to our knowledge. Thus the prediction of the model may lack critical physical elements, or it may be that the rotation brings unstable structures quickly into a stable orientation relative to the flow and the dispersion due to flow removes the effect before it is observable.

${ }^{1}$ J. Rabinow, AIEE Trans. 67, 1308 (1948).

${ }^{2}$ W. M. Winslow, J. Appl. Phys. 20, 1137 (1949).

${ }^{3}$ J. M. Ginder, in Encyclopedia of Applied Physics, edited by G. L. Trigg (VCH, Weinheim, 1996), p. 487.

${ }^{4}$ M. Parthasarathy and D. J. Klingenberg, Mater. Sci. Eng., R. 17, 57 (1996).

5 J. D. Carlson and J. L. Sproston, in Actuator 2000-Proceedings of the 7th International Conference on New Actuators, edited by H. Borgmann (Messe Bremen GmbH, Bremen, 2000), p. 126.

${ }^{6}$ D. J. Klingenberg, AIChE J. 47, 246 (2001).

${ }^{7}$ G. Bossis, E. Lemaire, J. Persello, and L. Petit, Prog. Colloid Polym. Sci. 89, 1 (1992).

${ }^{8}$ G. Bossis, Y. Grasselli, E. Lemaire, J. Persello, and L. Petit, Europhys. Lett. 25, 335 (1994).

${ }^{9}$ S. Henley and F. E. Filisko, J. Rheol. 43, 1323 (1999).

${ }^{10}$ O. Volkova, S. Cutillas, and G. Bossis, Phys. Rev. Lett. 82, 233 (1999).

${ }^{11}$ S. Vieira, L. B. Pompeo Neto, and A. C. F. Arruda, J. Rheol. 44, 1139 (2000).

${ }^{12}$ Y. Baxter-Drayton and J. F. Brady, J. Rheol. 40, 1027 (1996).

${ }^{13}$ J. R. Melrose and D. M. Heyes, J. Chem. Phys. 98, 5873 (1993).

${ }^{14}$ J. E. Martin, Phys. Rev. E 63, 011406 (2000).

${ }^{15}$ K. von Pfeil, M. D. Graham, D. J. Klingenberg, and J. F. Morris, Phys. Rev. Lett. 88, 188301 (2002).

${ }^{16}$ J. F. Morris and F. Boulay, J. Rheol. 43, 1213 (1999).

${ }^{17}$ P. R. Nott and J. F. Brady, J. Fluid Mech. 275, 157 (1994).

${ }^{18}$ R. H. Davis and A. Acrivos, Annu. Rev. Fluid Mech. 17, 91 (1985).

${ }^{19}$ Y. M. Shkel and D. J. Klingenberg, J. Appl. Phys. 83, 7834 (1998).

${ }^{20}$ P. Lorrain and D. R. Corson, Electromagnetic Fields and Waves, 2nd ed. (Freeman, New York, 1970).

${ }^{21}$ J. W. Cahn, J. Chem. Phys. 42, 93 (1965).

${ }^{22}$ J. A. Stratton, Electromagnetic Theory (McGraw-Hill, New York, 1941).

${ }^{23}$ M. C. Cross and P. C. Hohenberg, Rev. Mod. Phys. 65, 851 (1993).

${ }^{24}$ G. Bossis, H. Clercx, Y Grasselli, and E. Lemaire, Int. J. Mod. Phys. B 8, 2747 (1994)

${ }^{25}$ W. B. Russel, D. A. Saville, and W. R. Schowalter, Colloidal Dispersions (Cambridge University Press, New York, 1989).

${ }^{26}$ J. F. Brady and J. F. Morris, J. Fluid Mech. 348, 143 (1997).

${ }^{27}$ J. W. Cahn and J. E. Hilliard, J. Chem. Phys. 28, 258 (1958).

${ }^{28}$ K. von Pfeil, M. D. Graham, J. F. Morris, and D. J. Klingenberg (unpublished).

${ }^{29}$ K. von Pfeil, M.S. thesis, University of Wisconsin, Madison, WI, 2002.

${ }^{30}$ Y. M. Shkel and D. J. Klingenberg, J. Rheol. 43, 1307 (1999).

${ }^{31}$ R. Aris, Vectors, Tensors and the Basic Equations of Fluid Mechanics (Prentice-Hall, Englewood Cliffs, NJ, 1962). 